\title{
Aerodynamic optimization of a biplane configuration using differential evolution
}

\author{
R. W. Derksen \& A. G. Kraj \\ Department of Mechanical and Manufacturing Engineering, \\ University of Manitoba, Winnipeg, Manitoba, Canada
}

\begin{abstract}
This paper presents our work on designing a biplane configuration that has a minimum drag to lift ratio. This problem is a mixed optimization problem in that both discrete and continuous variables are used. Fourteen parameters were used to fully describe the biplane configuration and calculate performance. Performance calculations were based on Munk's general biplane theory. Each wing required six parameters; airfoil profile type, span, tip and root chord lengths, angle of attack, and sweep angle. Two parameters were used to define the horizontal stagger and vertical gap between the two planes. The airfoil profile types were stored in an indexed database which allowed us to obtain the section's aerodynamic characteristics. Our analysis showed that differential evolution found the optimum solution quickly. The characteristics of the resultant optimum solution will be discussed in detail, along with our observations of how the process needs to be adjusted for optimum performance.
\end{abstract}

Keywords: aerodynamic design, optimization, biplanes, aerodynamic configuration.

\section{Introduction}

The following sections will provide a brief review of the state-of-the-art of aerodynamic optimization. This will be followed by a discussion of the advantages and disadvantages of the biplane configuration. The introductory comments will conclude with the motivation for doing this work.

\subsection{The practice of aerodynamic optimization}

A quest for performance has been a key component in the development of aviation from the start. A great deal of this was motivated by high performance 
needs for military requirements, but civilian aviation has also contributed with the need for less expensive operational costs.

The early difficulty of obtaining solutions to aircraft design problems such as the determination of aerodynamic performance resulted in compromises in the design process. The optimization of aircraft design was usually the result of coordinated but separate efforts; say structural optimization for weight and streamlining. The resulting design would be the negotiated and mutually acceptable compromise. The optimization of aircraft configurations has generally progressed incrementally by a process of continuous improvement, occasionally incrementally advanced by technological and scientific discovery. This article will focus only on aerodynamic issues.

One of the earliest known optimum aerodynamic solutions is Munk's [1] work demonstrating that a wing which has an elliptic lift distribution along its span has minimum induced drag. Similar developments were the development of the area rule and of the use of wing sweep to minimize drag. These methods were very useful in developing design targets but often conflicted with other criteria.

The parallel development of computational flow analysis methods, numerical optimization and computer technology resulted in a new interest in aerodynamic optimization. This is a relatively new field that was initiated during the 1970s and is well represented by the pioneering work of Liebeck [2]. This work was directed to the development of high lift airfoils and can be thought of as an inverse design problem, as we would seek to find the shape that best matched a prescribed pressure distribution. The prescribed pressure distribution was crafted to provide a Stratford [3] pressure distribution in the adverse pressure zone and hence avoid separation. The analytical methods were based on potential flow methods that did not explicitly consider viscous effects. While this will generally result in improved profiles they will not necessarily be optimum.

The work of Rogalsky et al [4] clearly demonstrated that a global optimizer was required for this type of problem as the solution space contains many minima, which can trap other methods. Additionally, Differential Evolution was demonstrated to be highly effective at finding the optimum. However, convergence was slow, and often required 50,000 flow field simulations.

Consideration of the effect of viscosity requires us to use the Navier-Stokes equations to model the flow. This presents serious problems on two fronts.

The first front is that the computational demands of a Navier-Stokes solution are sever. For example, Lombardi et al [5] demonstrated a typical evaluation of drag on an aerofoil would take approximately 70 minutes for the standard $k-\varepsilon$ turbulence model on a 500-Mhz Pentium III. If 50,000 flow field simulations were required as typical of an inviscid optimization, convergence would take 6.6 years of CPU time. This time could be significantly larger as the nonlinear effect of the viscosity on the solution space may require significantly more flow field simulations to obtain convergence of the optimization process.

One of the methods proposed for dealing with the computation complexity in multidisciplinary design for aerodynamic optimization has been the use of neural 
nets and fuzzy logic. Here the concept is to reduce the number of simulation by using existent solutions, as described by Hajela [6]. These methods appear to offer great value to improving the speed of an optimization, but more study is needed.

The second front is what is the most appropriate objective or cost function to use. While this question is very important to those seeking an optimum shape, it is outside of the mechanics of the optimization process.

An example of a more complex optimization process can be found in the work of Mailon et al [7] in their work on obtaining the optimum configuration of a subsonic lifting wing. This study considered the influence of both viscosity and compressibility and should be considered as a good representation of the state-of-the-art in aerodynamic optimization. Unfortunately, the authors still rely on a down-hill gradient based optimization method, which is characteristic of the work on viscous optimization.

One should note that nearly all of the work on aerodynamic optimization has been based on continuous spaces. The real world often requires us to consider mixed parameters that also have integer values such as the number of blades to use in a compressor, the number of flaps for a high lift wing, or discrete values such as material properties. No examples of aerodynamic optimizations using mixed parameters have been found in the literature.

\subsection{Biplanes}

The earliest successful aircraft were biplanes for the most part with relatively few examples of monoplanes and triplanes in existence. The predominant reason for selecting the biplane configuration was that it had inherent structural advantages and permitted structures of great strength relative to their weight. The early monoplanes tended to be frail and required substantial external wire bracing that resulted in high drag. Advances in structural design capabilities and the requirement for higher speed resulted in the virtual elimination of multi-plane configurations in the late 1930s. The last biplane design is the Soviet AN-2 Colt which was developed in the 1950s and is still in operation in some locations. No new biplane has been designed since that time.

There are advantages of the biplane configuration. Namely that light weight and strong structures are easier to obtain than for monoplanes. Another advantage is that for a given maximum wing span we have a higher effective aspect ratio with larger lifting area, which results in lower induced drag.

No modern designer would consider a biplane for most aircraft applications. However, if wing span was restricted and the known operational speeds were low this configuration could offer an attractive advantage.

\subsection{Motivation for this work}

The motivation for this work comes from our university's participation in the SAE Aerodesign contest. In this annual contest, teams of undergraduate students design and build a radio controlled airplane and compete with other teams to see 
who can carry the greatest payload weight, with a consideration of the quality of their engineering judged on their work and the resultant reports.

Each year, the contest specifies a number of rules that the design must conform to. Such as the make and model of engine that can be used, as well as its possible modifications, rules for the current competition can be found on the Society of Automobile Engineers web site [8].

This work comes from a year where the maximum wing span was limited to 6 feet, with no restriction on plan form area. As these aircraft operate at low speed it was postulated that a biplane might give the best performance, hence our interest in seeing if we could find the optimum configuration using Differential Evolution to optimize the design. A detailed discussion of this work can be found in Kraj [9].

\section{Problem specification}

The design of an optimum aircraft configuration is a very complex issue, where the determination of what performance factor to optimize is not always clear. This becomes clear when considering that the design of a stunt plane has different objectives than used in the design of a passenger plane.

The objective of the High-lift contest is to lift the maximum weight. Clearly, this will depend on aerodynamics (maximum lift and minimum drag), the structure (strength and weight of the airframe), aircraft stability, and so on. The integration of these areas is well beyond the scope of this project. We elected to examine a simpler problem of finding a configuration that minimizes the drag to lift ratio of the biplane configuration itself, neglecting the effect of fuselage, tail surfaces and other components.

\subsection{Configuration}

Assumed layout is that of two flying surfaces spaced apart by a vertical distance, the inter plane spacing $h$, with a horizontal stagger $s$. The stagger is defined at the signed horizontal distance between the leading edge of plane 1 to the leading edge of plane 2, where negative values of stagger imply that plane 2 is ahead of plane 1. Each flying surface has a simple trapezoidal layout with span $b$, root chord $C_{r}$ and tip chord $C_{t}$. It is assumed that the flying surfaces us a single airfoil profile inclined a constant angle of attack, $\alpha$. Additionally each plane's leading edge can be swept at an angle $\phi$. Notice that each of the each quantity is subscripted with the exception of the inter plane spacing and stagger. This is shown in Figure 1, below.

\subsection{Airfoil database}

The airfoil database used a very simplified airfoil model and employed data for a limited number of profiles to keep this work tractable. A total of five NACA airfoils were used in the database the NACA 1408, NACA 1412, NACA, 2412, NACA 2415, and NACA 4412 profiles. Each profile was numbered and had the 
following data associated with it, minimum and maximum permissible angles of attack, maximum and minimum lift coefficients, zero incidence lift coefficient, the lift curve slope, maximum and minimum pitching coefficient, zero incidence pitching coefficient, the pitching curve slope and finally the zero incidence drag coefficient. For the purposes of this exercise it was assumed that the lift and moment curves were linear between the minimum and maximum permissible angles of attack.

The resulting optimum biplane configuration is limited by the restricted data set. However, it is believed that this work is a fully representative example of a more exhaustive study.
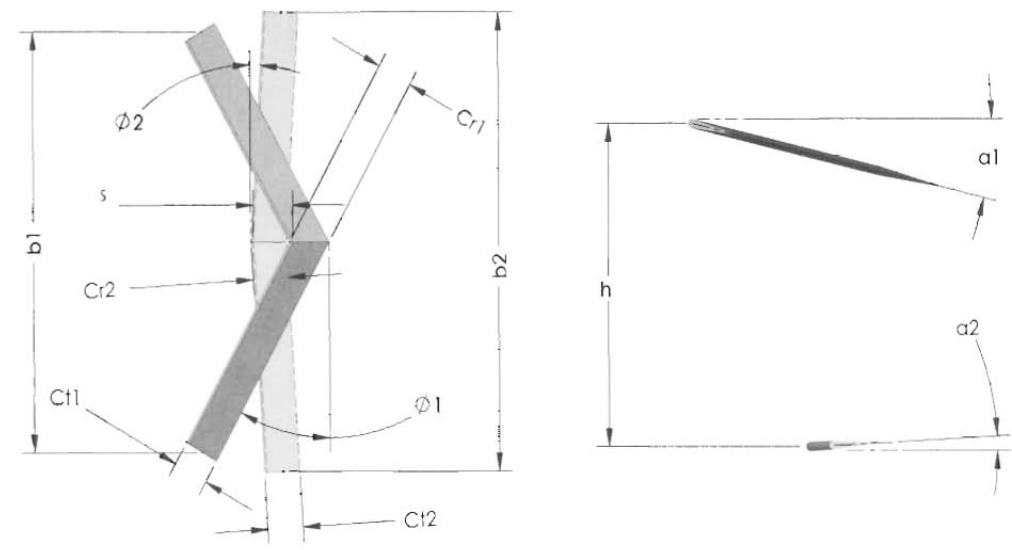

Figure 1: The generic biplane configuration and specified geometric parameters used in this study.

\subsection{The problem parameters}

There were a total of 14 design parameters that were to be optimized. Two of the parameters, the profiles for each wing, are discreet variables, and hence are not differentiable. The 12 remaining parameters are all continuous parameters. This yields a non-differentiable optimization problem with is not amenable to any of the gradient based optimization methods, and is an interesting application for those interested in mixed parameter optimization.

\section{Aerodynamic modelling: Munk's biplane theory}

Max Munk's work on determining the aerodynamic forces on biplane configuration was presented by NACA in 1923 [10]. His work addressed the problem of two-dimensional flow over a biplane configuration neglecting viscosity. His study examined the influence of airfoil section, chord, gap, stagger, the incidence of the individual planes, and the influence of the lateral dimensions. This work also considered the work of earlier investigations to 
include the effect of viscosity and to utilize existing experimental experience. He simplified the method for practical application, where simple formulas were given to obtain the drag, lift, and moment for an arbitrary configuration. Munk's work still represents the state-of-the art for work on biplanes and has proven to be quite successful. As such, we have adopted it for use in our work with out modification. Once the design parameters are specified it was a simple problem to calculate the configuration's performance through a relatively simple set of equations.

\section{Optimization}

The resultant configuration was required to satisfy the 2003 SAE Aerodesign competition. The relevant rule is that the total span of all planes be less that 6.0 $\mathrm{ft}$., while there was no limitation on total plan form area. The flow conditions were taken to be standard temperature and pressure. All calculations employed an assumed airspeed of $35 \mathrm{mph}$ based on previous contest experience with the permitted engine.

The purpose of this optimization project was to find the biplane configuration with the minimum drag to lift ratio as it was assumed that this would result in the best performance. One should be cautious of this suggestion as many other factors could result in better performing aircraft than the configuration obtained here. These factors include the installed thrust of the engine, the necessary structural weight, the influence of other components such as the fuselage, and other factors. These factors would have a direct impact on both the empty weight and operational speed.

\subsection{Differential evolution}

Differential Evolution is a genetic algorithm developed by Storn and Price $[11,12]$. This method is not based on bit-flipping, but rather bases the evolutionary process on the population of candidate solutions. The advantage of this is that the method adapts to the solution space and is generally much faster. In this method an initial population of candidate solutions that spans the solution space of randomly selected members is created. The population sized is typically selected to be ten times the number of the parameters, 140 for this work. A child candidate is then created for each member of the parent generation by mutating it and recombining it with another. Then a new generation can be constructed by selecting between the child and parent that is fittest. Storn and Price have developed several ways to perform these operations. The specific method used in this work is $\mathrm{DE} /$ best/1/exp with $\mathrm{F}=0.9$ and $\mathrm{K}=1.0$. This process would be repeated until the minimum was found or until the $800^{\text {th }}$ generation.

\subsection{Dealing with unrealistic population members: constraint violations}

The nature of this problem required us to place constraints on the parameters such as the maximum limit on the span being less than $6 \mathrm{ft}$. Examples of the other constraints include the following. The index of the airfoil type had to be 
constrained to reflect that this study only used five airfoils. The angle of incidence of the wing had to be within the range of the available data. Additionally, the configuration of the wings could not interfere.

A child population member is created without regard to these constraints in mind. We dealt with constraint violations in two different ways, by using a penalty method and by adjusting the child population member to satisfy the constraint.

\subsubsection{Method 1: rejecting the population member using the penalty method}

The penalty method is the easiest to employ and describe. No consideration was given to adjusting the population member that violates the constraint. However, if a constraint was encountered, the value of its objective function was set to its largest possible value. This results in it automatically losing the tournament with its parent and subsequent rejection.

\subsubsection{Method 2: retaining the population member and adjusting the parameter causing the constraint violation}

In this method the variable that is causing the constraint violation was set to the value of the closest value of the constraint. The result is an acceptable candidate, however, the impact on the optimization process is hard to determine. One could argue that the variable should be set to a random value that does not violate the constraint could also be successful.

\section{Resultant designs}

The resultant designs are given in the following subsections. Both designs are quite novel, and atypical of common biplane designs.

\subsection{Method 1 results}

Resulting design using Method 1 resulted in a configuration with a drag to lift of $3.09 \times 10^{-2}$, and is shown below. Convergence was obtained in 76 generations, and the computed drag was $0.1923 \mathrm{lbf}$ and lift was $6.22 \mathrm{lbf}$.

The configuration resulted in a vertical gap between planes of 24 ins, and the leading edge of the upper plane at the root being 6.5 ins behind the leading edge of the lower plane at the root. The upper plane is a NACA 4412 airfoil section set at an angle of attack of $13^{\circ}$. Its plan form has a constant chord length of 6 ins and span of $6 \mathrm{ft}$, with $30^{\circ}$ forward sweep. The lower plane is also a NACA 4412 airfoil section set at an angle of attack of $-2.8^{\circ}$. Its plan form also has a constant chord length of 6 ins and span of $6 \mathrm{ft}$, but with $3.5^{\circ}$ backward sweep.

\subsection{Method 2 results}

Resulting design using Method 2 resulted in a configuration with a drag to lift of $2.79 \times 10^{-2}$, and is shown below. Convergence required 600 of the total possible 800 generations, and the computed drag was $0.1135 \mathrm{lbf}$ and lift was $4.07 \mathrm{lbf}$. 


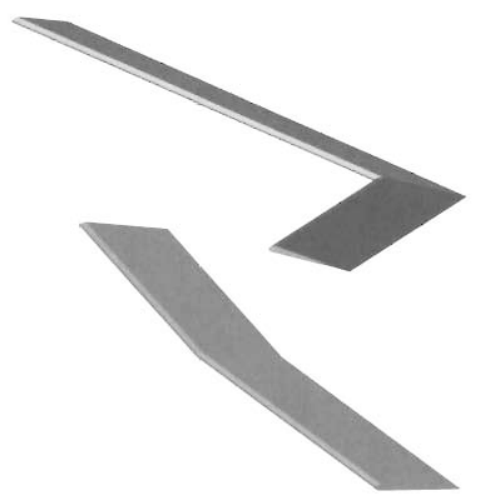

Figure 2: $\quad$ The final biplane configuration using Method 1.
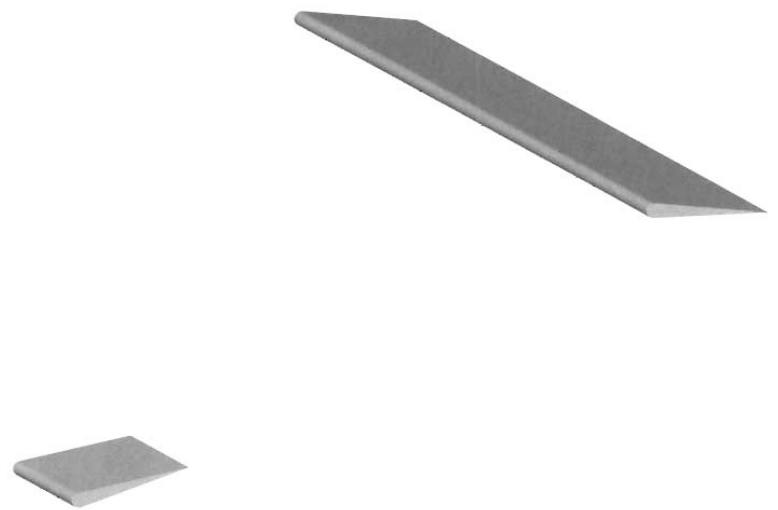

Figure 3: $\quad$ The final biplane configuration using Method 2.

The configuration resulted in a vertical gap between planes of 15 ins, and the leading edge of the upper plane at the root being 24 ins behind the leading edge of the lower plane at the root. The upper plane is a NACA 4412 airfoil section set at an angle of attack of $13^{\circ}$. Its plan form has a constant chord length of 6 ins and span of $6 \mathrm{ft}$, without sweep. The lower plane is a NACA 1408 airfoil section set at an angle of attack of $-6^{\circ}$. Its plan form has a constant chord length of 6 ins and span of $1 \mathrm{ft}$, without sweep.

\section{Discussion and conclusions}

The original expectation was that both methods should result in the same optimum design, as the solution space was not changed. Our results did not show this as both designs were radically different. Method 1 favoured swept 
wings of roughly the same area, while Method 2 favoured straight wings with a canard with a small leading wing. Additionally, Method 2 resulted in a lower drag to lift ratio than Method 1. This appears to indicate that adjusting parameters that violate a constraint is the better method for dealing with constraint violations. The reason for this is that the boundaries of the solution space are much more thoroughly searched as each constraint violation resulted in a population member located on the boundary of the design space. Some support for this could be evident in that Method 2 was significantly slower to converge than Method 1 by a factor of roughly 8 to 1 . This should not be a factor if the optimum is an interior point in the design space.

Our general observation on the use of Differential Evolution for the optimization of a biplane is that it was very quick, even in the worst case, and is very easy to employ. It is clearly a good candidate method for finding optimum aerodynamic configuration.

\section{Acknowledgements}

The authors would like to acknowledge the support of the Natural Sciences and Engineering Research Council of Canada through a grant held by R.W. Derksen.

\section{References}

[1] Munk, M.M., Isoperimetriche Probleme aus der Theorie des Fluges, Göttingen Dissertation, 1918.

[2] Liebeck, R.H., A Class of Airfoils Designed for High Lift in incompressible Flow. Journal of Aircraft, 10(10), pp. 610-617, 1973.

[3] Stratford, B.S., An Experimental Flow With Zero Skin Friction Throughout its Region of Pressure Rise. Journal of Fluid Mechanics, 5, pp. 17-35, 1959.

[4] Rogalsky T., Kocabiyik S., and Derksen R.W., Differential Evolution in Aerodynamic Optimization. Canadian Aeronautics and Space Journal, 46(4), pp. 183-190, 2000.

[5] Lombardi, G., Salvetti, M.V., and Pinelli, D., Numerical Evaluation of Airfoil Friction Drag. Journal of Aircraft, 37(2), pp. 354-356, 2000.

[6] Hajela, P., Soft Computing in Multi-Disciplinary Aerospace Design New Directions for Research. Progress in Aerospace Sciences, 38, pp 121, 2002.

[7] Mialon, B., Fol, T, and Bonnaud, C., Aerodynamic Optimization of Subsonic Flying Wing Configurations. American Institute for Aeronautics and Astronautics, paper AIAA-2002-2931, 2002.

[8] Society of Automobile Engineers, http://students.sae.org/competitions/ aerodesign/

[9] Kraj, A.G., Aerodynamic Optimization of a Biplane Configuration through Differential Evolution, B.Sc. (Mech Eng.) Thesis, University of Manitoba, Winnipeg, Manitoba, 2003. 
218 Computer Aided Optimum Design in Engineering X

[10] Munk M.M., General Biplane Theory, National Advisory Committee for Aeronautics, NACA-TR-151, 1923.

[11] Storn, R. and Price, K., Differential Evolution. Dr. Dobb's Journal, 22(4), pp. 18-24, 1997.

[12] Storn, R. and Price, K., Differential Evolution - a Simple and Efficient Heuristic for Global Optimization over Continuous Spaces. Journal of Global Optimization, 11(4), pp. 341-359, 1997. 